\title{
Post-Stop-Signal Adjustments: Inhibition Improves Subsequent Inhibition
}

\author{
Patrick G. Bissett and Gordon D. Logan \\ Vanderbilt University
}

\begin{abstract}
Performance in the stop-signal paradigm involves a balance between going and stopping, and one way that this balance is struck is through shifting priority away from the go task, slowing responses after a stop signal, and improving the probability of inhibition. In 6 experiments, the authors tested whether there is a corresponding shift in priority toward the stop task, speeding reaction time to the stop signal. Consistent with this hypothesis, stop-signal reaction time (SSRT) decreased on the trial immediately following a stop signal in each experiment. Experiments 2-4 used 2 very different stop signals within a modality, and stopping improved when the stop stimulus repeated and alternated. Experiments 5 and 6 presented stop signals in different modalities and showed that SSRT improved only when the stop stimulus repeated within a modality. These results demonstrate within-modality post-stop-signal speeding of response inhibition.
\end{abstract}

Keywords: cognitive control, inhibition, post-stop-signal slowing, sequential control adjustments, stopsignal paradigm

Cognitive control is required to balance the demands of the ever-changing environment. One common task used to investigate cognitive control is the stop-signal paradigm (Lappin \& Eriksen, 1966; Logan \& Cowan, 1984), which directly pits a go task against a stop task. Performance in the go task requires speed, and performance in the stop task requires caution. Control adjustments are necessary to achieve a balance between going and stopping, and one common adjustment is the slowing of go reaction time (RT) after a stop signal occurs (Bissett \& Logan, 2011a; Emeric et al., 2007; Rieger \& Gauggel, 1999; Verbruggen, Logan, Liefooghe, \& Vandierendonck, 2008). One explanation for post-stop-signal slowing is that it is a goal-priority shift away from the go task (Bissett \& Logan, 2011a). Slowing of go RT will improve subjects' ability to stop, increasing their probability of inhibition. But this does not mean that the stop process itself is affected. In six experiments, we examine whether the priority shift away from the go task after stop trials is coupled with a priority shift toward the stop task, measured in faster stop-signal reaction time (SSRT).

Several studies suggest that successive stop trials may slow SSRT. Van den Wildenberg, van der Molen, and Logan (2002) had subjects prepare for a no-go response and found an increase in SSRT during the preparatory period. Bissett, Nee, and Jonides (2009) also found an increase in SSRT following a visual stimulus that predicted a no-go trial. Verbruggen and colleagues found slower SSRT on trials that required inhibiting incompatible responses in Stroop and flanker (Verbruggen, Liefooghe, \& Vand-

This article was published Online First January 23, 2012.

Patrick G. Bissett and Gordon D. Logan, Department of Psychology, Vanderbilt University.

This research was supported by Grant BCS-0957074 from the National Science Foundation.

Correspondence concerning this article should be addressed to Patrick G. Bissett or to Gordon D. Logan, both at Department of Psychology, Vanderbilt University, Nashville, TN 37240. E-mail: patrick.g.bissett@vanderbilt.edu or gordon.logan@vanderbilt.edu ierendonck, 2004) and Simon tasks (Verbruggen, Liefooghe, Notebaert, \& Vandierendonck, 2005). However, Morein-Zamir, Chua, Franks, Nagelkerke, and Kingstone (2007) found faster SSRT in a task that required subjects to stop applying a constant pressure if they had also stopped applying pressure on the previous trial. The critical difference between the studies showing slower and faster SSRTs may lie in the similarity of the stop tasks and stop signals. Studies that found slower SSRT compared different tasks and different stop stimuli, whereas the study that found faster SSRT compared the same task with the same stop signal. This motivates the evaluation of stimulus differences and modality differences in the present study. Morein-Zamir et al. (2007) used an unusual stop task, which motivates the investigation of more typical stop tasks in the present study.

\section{Stop-Signal Paradigm}

In the stop-signal paradigm, subjects typically perform a choice RT task (the "go" task) and are asked to withhold their response when a stop signal occurs on a random subset of trials. The delay between the go stimulus presentation and the stop signal (stopsignal delay, or SSD) is adjusted to manipulate the probability of inhibition. When SSD is short, subjects frequently inhibit their responses, but as SSD increases, the probability of inhibition decreases. These findings have been explained with the "horse race" model (Logan \& Cowan, 1984), which assumes that a go process, initiated by go stimulus onset, races against a stop process, initiated by stop-signal onset. If the go process finishes before the stop process, subjects fail to inhibit, producing a signalrespond trial. If the stop process finishes before the go process, subjects succeed at inhibiting, producing a signal-inhibit trial. Stop-signal delay biases the race between stop and go processes: Short SSDs bias the race in favor of stopping and thereby increase the probability of inhibiting; long SSDs bias the race in favor of going and thereby increase the probability of responding. SSD is often adjusted with a staircase procedure, increasing after signalinhibit trials and decreasing after signal-respond trials to yield a 
probability of inhibition of .5 (Levitt, 1971), which means that the race between going and stopping is tied (Logan, Schachar, \& Tannock, 1997).

\section{Sequential Control Adjustments and Stopping}

The research on sequential control adjustments in stopping fits into a larger framework of sequential adjustments in cognitive control. Three main theoretical frameworks for sequential adjustments are error monitoring (Laming, 1968; Rabbitt, 1966), conflict monitoring (Botvinick, Braver, Barch, Carter, \& Cohen, 2001; Carter et al., 1998), and episodic memory retrieval (Verbruggen \& Logan, 2008a, 2008b). The error monitoring hypothesis suggests that subjects slow their responses after errors by increasing their response threshold (Rabbitt, 1966), strategically trading speed for accuracy to reduce the probability of future errors. This explanation has been challenged by research that showed that accuracy does not always increase after errors (Gehring, Goss, Coles, Meyer, \& Donchin, 1993; Hajcak \& Simmons, 2008; Rabbitt \& Rodgers, 1977). In the stop-signal paradigm, some research has shown greater slowing after stop errors (Rieger \& Gauggel, 1999; Schachar et al., 2004; Verbruggen et al., 2008), but some has not (Bissett \& Logan, 2011a; Emeric et al., 2007). Stop and go accuracy do not increase or decrease consistently after stop errors (Bissett \& Logan, 2011a). It remains an open question whether errors in stopping are different from other errors. Most error monitoring studies address errors of choice, which may be different from errors of timing (i.e., going too fast) in the stop-signal paradigm (Bissett \& Logan, 2011b).

The conflict monitoring hypothesis suggests that coactivation of competing responses recruits control to increase the likelihood of achieving task goals. The conflict hypothesis addresses slowing after errors but also addresses other control adjustments, like focusing attention in the Stroop and flanker tasks (Botvinick et al., 2001). Schall and colleagues have applied the conflict framework to countermanding eye movements in monkeys (Emeric et al., 2007; Ito, Stuphorn, Brown, \& Schall, 2003; Stuphorn \& Schall, 2006; Stuphorn, Taylor, \& Schall, 2000). They measured conflict as coactivation of movement and fixation neurons, and because fixation and movement neurons are both active on successful inhibition trials, they suggested that post-stop-signal slowing should be greatest after successful inhibition. This result is not generally found (Bissett \& Logan, 2011a; Rieger \& Gauggel, 1999; Schachar et al., 2004; Verbruggen et al., 2008), and their own results that led them to this conclusion (Emeric et al., 2007) were likely an artifact of using an inappropriate baseline (Nelson, Boucher, Logan, Palmeri, \& Schall, 2010). This is strong evidence against Schall and colleagues' conceptualization of conflict in the stop-signal paradigm, but other conceptualizations are possible. For example, the race model (Logan \& Cowan, 1984) suggests that go and stop processes are both active on all stop-signal trials, suggesting post-stop-signal slowing after both successful and failed inhibition.

Episodic retrieval hypotheses suggest that when an item occurs, many things are stored with it, like the context it appeared in, the goals that were active, and the response made to it (Barsalou, 1990; Logan, 1988; Tulving, 1972). There are several episodic retrieval hypotheses in the literature that address many different phenomena (Altmann, 2011; Barsalou, 1990; Hommel, 1997;
Hommel, Proctor, \& Vu, 2004; Logan, 1988, 1990; Mayr, Awh, \& Laurey, 2003; Medin \& Schaffer, 1978; Neill \& Valdes, 1992; Neill, Valdes, Terry, \& Gorfein, 1992; Nosofsky, 1984; Tulving, 1972; Verbruggen \& Logan, 2008a, 2008b). The theories differ in detail, but they all predict that retrieval will be faster and more likely when episodes are the same than when they are different. We test this prediction in Experiments $2-6$ by varying the similarity between successive stop signals.

Several studies show that episodic retrieval is important in post-stop-signal processing. Verbruggen et al. (2008) found greater slowing when the go stimulus repeats and suggested that go stimuli are associated with stopping. These associations are retrieved when the go stimulus repeats, and the retrieved associations slow go RT (also see Lenartowicz, Verbruggen, Logan, \& Poldrack, 2011; Verbruggen \& Logan, 2008a, 2008b). Stopping may also be associated with concurrent stimuli that are irrelevant to the task (Kühn \& Brass, 2009; Kühn, Elsner, Prinz, \& Brass, 2009).

\section{The Present Experiments}

In the present study, we investigated the effect of stopping on subsequent stopping performance. In Experiment 1, like many stop-signal experiments, we used a single auditory stop signal that occurred on a random $40 \%$ of all trials. Additionally, we waited to adjust SSD until after any set of multiple stop signals was complete, yielding the same SSD for all stop signals in a set. Experiment 2 replicated Experiment 1, except we randomly interleaved two different auditory stop signals to test the degree to which the improvement in SSRT we observed in Experiment 1 was stimulus specific. Experiments 3 and 4 replaced the two auditory stop signals of Experiment 2 with two visual stop signals to test the generalizability of the stop-signal repetition effect to the visual modality and to test the effects of having the go stimulus and stop signal in the same modality (visual). Experiments 5 and 6 interleaved one visual and one auditory stop signal. In Experiments 2, 3 , and 4 , the two stop signals were very different from each other but were presented within the same modality. If improvement in SSRT depends on similarity, as the episodic retrieval hypotheses suggest, we should see greater improvement for repetitions than for alternations; if improvement in SSRT does not depend on similarity, then we should see equivalent improvement for repetitions and alternations. Experiments 5 and 6 used different stimuli in different modalities to assess the role of modality differences in SSRT improvements. If improvement in SSRT depends on stimulus modality, then we should see greater improvement for modality repetitions than for alternations; if improvement in SSRT does not depend on stimulus modality, then we should see equivalent improvement for modality repetitions and alternations. Together, these experiments tested whether shifting goal priority away from the go task after stop trials is coupled with shifting priority to the stop task: Is post-stop-signal slowing coupled with faster SSRT?

\section{Experiment 1}

\section{Method}

Subjects. Twenty-four subjects were recruited from the Nashville community and were compensated \$12 for a single 1-hr 
session. All subjects had normal or corrected-to-normal vision. We replaced three subjects for mean accuracy below $85 \%$ and one subject for unusually slow RT ( $>3 S D$ s above the mean).

Apparatus and stimuli. The experiment was run on a Pentium Dual-Core PC running E-Prime 1 (http://www.pstnet.com). The stimuli were presented on a $48-\mathrm{cm}$ cathode ray tube monitor. The go task was to respond to a single black shape on a white background presented in the center of the screen. The shape was chosen from a set of four shapes: triangle, circle, square, or diamond. The height and width of each shape were $4 \mathrm{~cm}$ at the longest point. Subjects responded by pressing $z$ or $m$ on a QWERTY keyboard with the left or right index finger, respectively. The stop signal was a $500-\mathrm{Hz}$ tone $(70 \mathrm{~dB}, 100 \mathrm{~ms})$ presented through closed headphones (Sennheiser eH 150).

Procedure. Each trial began with a 500-ms fixation display, followed by the go stimulus. The go stimulus remained on the screen for $850 \mathrm{~ms}$. It was followed by a 1,000-ms intertrial interval during which the screen was blank.

The stop signal indicated to subjects that they should withhold their response for that trial. Stop signals occurred on a random $40 \%$ of the trials. The delay between the presentation of the go stimulus and the stop signal (stop-signal delay, or SSD) was varied according to a tracking procedure designed to achieve a probability of responding of .5 on isolated stop signals and first stop trials in sequences of two or more (Levitt, 1971). In order to compare the first and second stop signals in sequences of two or more as directly as possible, we did not adjust SSD until the sequence of two or more was complete. Based upon stop success or failure on the first stop signal in sequences of two or more, SSD was increased (success) or decreased (failure) by $50 \mathrm{~ms}$ after the sequence was complete. This resulted in equal SSDs across each stop trial in sequences of two or more. This also meant that stop success may not equal .5 on stop trials after the first in sequences of two or more, as SSD adjustment was not based upon stop outcome on these trials. Because different rates of stop success may occur on stop trials of interest, we used the integration method to calculate SSRT (Logan \& Cowan, 1984).

The experiment began with written and verbal instructions. Subjects were instructed to respond quickly and accurately to the shape and to do their best to withhold their response when stop signals occurred. Two of the shapes were mapped onto each response. Response mapping was counterbalanced across subjects. We collapsed across repetitions and alternations of the go stimulus and correct go response in the data analyses. Subjects were told not to wait for the stop signal. After the instructions, subjects were given 24 trials of experimenter-supervised practice. After practice, subjects completed the main task, which included five blocks of 240 trials. At the end of each block, subjects were given feedback on mean RT and mean accuracy from that block.

\section{Results}

We first evaluated the results without examining sequential effects. Isolating correct trials without a stop signal, or nosignal trials, revealed that mean no-signal RT was $650 \mathrm{~ms}$ and mean no-signal accuracy was $96 \%$. Mean probability of a making a response given a stop signal, or $p$ (respondlsignal), was .44. This value was below $50 \%$ because this incorporates $p$ (respondlsignal) values of stop-signal sequences of two or more, but SSD changed only based on the first stop signal in the sequence. If subjects slow after a stop signal, they will improve their ability to stop on an immediately following stop signal, decreasing the $p$ (respondlsignal). But this does not mean that SSRT must improve; if go RT increases and SSD and SSRT remain the same, $p$ (respondlsignal) will decrease. We calculated $p$ (respondlsignal) for only the first stop-signal trials in any sequence (i.e., those trials that affected the SSD tracking algorithm) and found a value very close to $.5(M=.49)$. Mean SSD was $409 \mathrm{~ms}$. The ordering of the RT distributions for signalrespond and go trials confirms the predictions of the race model (see Appendix, Figure A1a) and justifies our use of the race model to estimate SSRT.

Our analyses focused on stopping performance on the first (S1) and second (S2) trials in stop trial sequences of two or more. To reveal post-stop-signal slowing, we would expect RTs that followed one stop signal $(\mathrm{S}+1 \mathrm{RT})$ to be slower than RTs that followed at least one go signal $(\mathrm{G}+1 \mathrm{RT})$, which is what we found $(M \mathrm{~S}+1 \mathrm{RT}=656 \mathrm{~ms}, M \mathrm{G}+1 \mathrm{RT}=634 \mathrm{~ms}), t(23)=$ $4.04, p<.01 .^{1}$ This slowing indicates a shift in priority away from the go task. To determine whether there was also a shift in priority toward the stop task, we asked whether SSRT was faster on the second stop trial than on the first.

We calculated SSRT with the integration method (Logan \& Cowan, 1984), which involves estimating the point in time at which the stop process finishes from the go RT distribution from no-stop-signal trials and the probability of responding given a stop signal (i.e., $p$ [respondlsignal]). According to the race model, go RTs faster than the point at which the stop process finishes will escape inhibition, producing a signal-respond trial, and go RTs slower than this point will be inhibited, producing a signal-inhibit trial. We can estimate this point by calculating the percentile of the go RT distribution from no-stop-signal trials that corresponds to $p$ (respondlsignal), which yields the finishing time of the stop process relative to the onset of the go stimulus. We estimate SSRT relative to the onset of the stop signal by subtracting SSD from this percentile RT.

To examine sequential effects in SSRT, we had to use different no-stop-signal distributions for the first and second stop signal in a sequence because go RTs were slower after the first stop signal in a sequence. To calculate SSRT for the first trial in the sequence, we used the distribution of no-signal trial RTs that did not immediately follow a stop trial $(G+1)$. To calculate SSRT for the second trial in the sequence, we used the distribution of no-signal RTs that followed a single stop signal $(\mathrm{S}+1)$. We then calculated the $p$ (respondlsignal) separately for the first $(\mathrm{S} 1, M=.49)$ and second (S2, $M=.37$ ) stop signal in sets of two or more, which differed significantly, $t(23)=6.83, p<.01$. Next we estimated the RT from each distribution $(\mathrm{G}+1$ and $\mathrm{S}+1)$ at the percentile that

\footnotetext{
${ }^{1}$ Post-stop-signal slowing should be calculated as a single difference score $(S+1)-(S-1)$ if one wants to compare slowing after signalinhibit and signal-respond trials (Nelson et al., 2010). Post-stop-signal slowing may be calculated as a double difference score $[(S+1)-(S-$ $1)]-[(G+1)-(G-1)]$ if one wants to also compare go stimulus repetitions and alternations (Bissett \& Logan, 2011a). We do not make these comparisons, and the results are similar if calculated in these other ways, so we do not include these additional analyses.
} 
corresponded to $p$ (respondlsignal) for S1 and S2. Finally, we subtracted mean SSD (410 ms, which is the same for S1 and S2) from the RT at that percentile to yield SSRT. We found that SSRT for S2 $(M=184 \mathrm{~ms})$ was considerably faster than for S1 $(M=205$ $\mathrm{ms}), t(23)=4.32, p<.01$. This shows that subjects stop more efficiently if the previous trial was a stop trial (see Figure 1a), suggesting that stop signals shift priority away from the go task, toward the stop task.

\section{Experiment 2}

In Experiment 1, we found that SSRT improved on consecutive stop trials. We used only one stop signal (500-Hz tone), so all stop trial repetitions were also stop stimulus repetitions. To test whether the improvement in SSRT observed in Experiment 1 was driven by stop stimulus repetition, we used two very different auditory stopping tones in Experiment 2. This manipulation decoupled stop trial repetitions from stop stimulus repetitions, allowing us to test whether stop stimulus repetition may account for the Experiment 1 results.

\section{Method}

Subjects. Twenty-four subjects were selected and compensated as in Experiment 1. We replaced three subjects for accuracy below $85 \%$, two subjects for unusually slow RT ( $>3$ SDs above the mean), and one subject whose probabilities of successful stopping (calculated from those trials that contribute to the SSD a

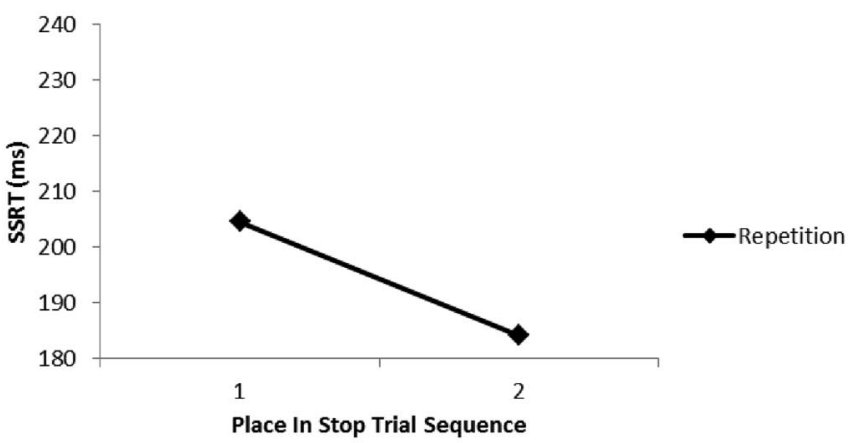

c

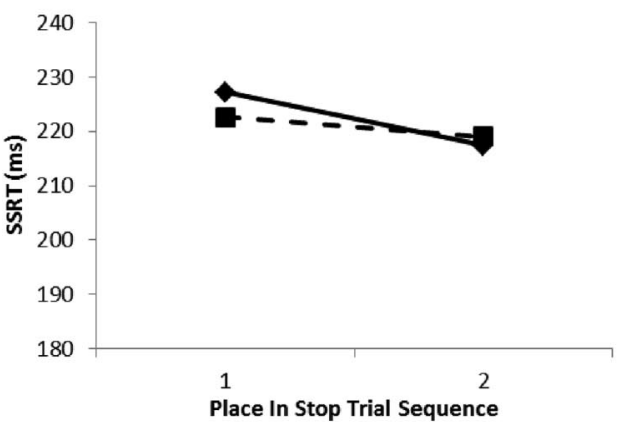

e

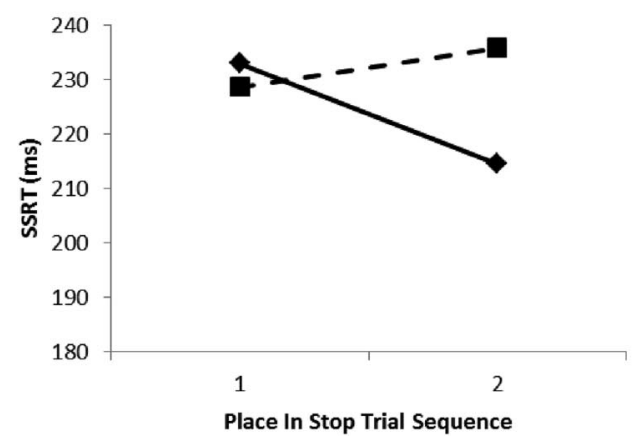

b Experiment 2 Sequential SSRT

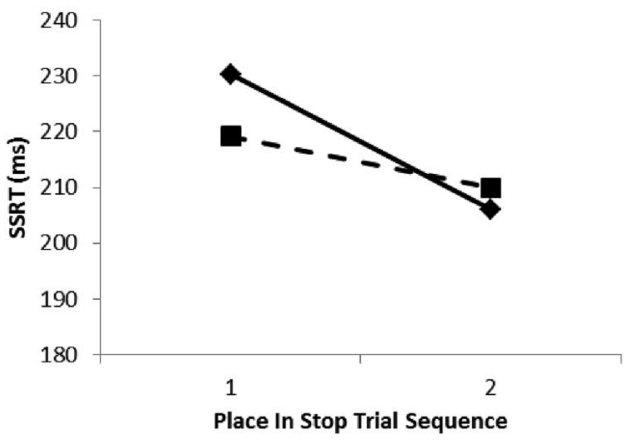

d

Experiment 4 Sequential RT

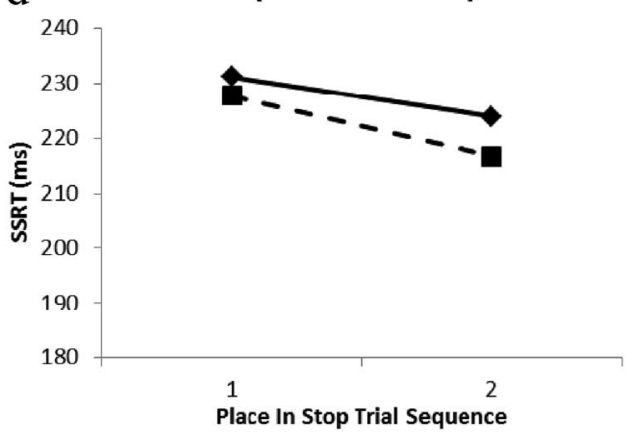

f

Experiment 6 Sequential SSRT

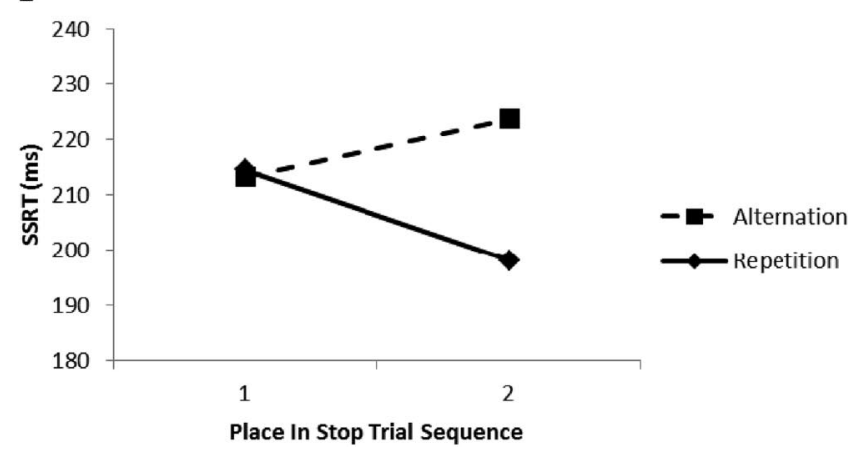

Figure 1. For Experiments 1-6 (Panels a-f, respectively), the stop-signal reaction times (SSRTs) in the first and second stop trials in stop trial sequences of two or more. In Panels b-f, SSRT is also separated by whether the two stop stimuli were the same (repetition) or different (alternation). 
tracking algorithm) fell outside the $95 \%$ confidence interval of .5 probability of stopping to a stop signal.

Apparatus and stimuli. The apparatus and stimuli were the same as in Experiment 1, with the exception that two auditory stop signals were randomly interspersed within the task. One was the same $500-\mathrm{Hz}$ tone $(70 \mathrm{~dB}, 100 \mathrm{~ms})$ used in Experiment 1 , and the other was a $750-\mathrm{Hz}$ tone $(70 \mathrm{~dB}, 100 \mathrm{~ms})$. The just noticeable difference in frequency in this range is somewhere between $1 \mathrm{~Hz}$ and $4 \mathrm{~Hz}$ (Shower \& Biddulph, 1931; Wever \& Wedell, 1941; Zwicker \& Fastl, 1990), so this difference in frequency is easily noticeable and large.

Procedure. The basic trial structure and instructions for Experiment 2 were identical to those of Experiment 1 with the following exception: On the $40 \%$ of trials in which a stop signal occurred, half of the stop signals were the $500-\mathrm{Hz}$ tone and the other half were the $750-\mathrm{Hz}$ tone.

\section{Results}

We first evaluated the results without examining sequential effects. Mean correct no-signal RT was $602 \mathrm{~ms}$, and mean nosignal accuracy was 94\%. Mean $p$ (respondlsignal) was .45 (see Experiment 1 results for an explanation for the value below .50). Additionally, $p$ (respondlsignal) did not significantly differ between the two tones $(500 \mathrm{~Hz} M=.45,750 \mathrm{~Hz} M=.45), t(23)<1, p>$ .4. We calculated $p$ (respondlsignal) for only those trials that affected the SSD tracking algorithm and found a value very close to $.50(M=.50)$. Mean SSD was $359 \mathrm{~ms}$. The ordering of the RT distributions for signal-respond and go trials confirms the predictions of the race model (see Appendix, Figure A1b) and justifies our use of the race model to estimate SSRT.

As in Experiment 1, our analyses focused on stopping performance on the first and second trials in stop trial sequences of two or more. Unlike in Experiment 1, we could now separate trials by whether the stop stimulus repeated or alternated in the two-trial sequence, testing whether the SSRT improvement in Experiment 1 was stimulus specific. For the SSRT calculations, we used separate SSD values and $p$ (respondlsignal) for repetition and alternation sequences. As in Experiment 1, the underlying go RT distributions differ between the first (S1, which uses the $\mathrm{G}+1$ go RT distribution) and the second ( $\mathrm{S} 2$, which uses the $\mathrm{S}+1$ go RT distribution) stop trial in a sequence because of post-stop-signal slowing. The underlying go RT distributions cannot be separated by stop stimulus repetition or alternation (i.e., no-signal trials cannot be categorized as stop stimulus repetitions or alternations), so the same go RT distributions were used for alternations and repetitions.

The results show that $\mathrm{S}+1 \mathrm{RT}(617 \mathrm{~ms})$ was significantly slower than $\mathrm{G}+1 \mathrm{RT}(589 \mathrm{~ms}), t(23)=6.07, p<.01$, revealing post-stop-signal slowing. There was no significant difference between SSDs in repetition sequences $(M=354 \mathrm{~ms})$ and alternation sequences $(M=360 \mathrm{~ms}), t(23)=0.92, p>.35$. The mean SSRTs are presented in Figure 1b. We evaluated the effect of place in sequence (S1 or S2) and stop stimulus repetition or alternation on SSRT using a $2 \times 2$ analysis of variance (ANOVA; see Figure $1 \mathrm{~b}$ ), which revealed that S2 SSRT $(M=208 \mathrm{~ms})$ was faster than S1 SSRT $(M=225 \mathrm{~ms}), F(1,23)=10.46, M S E=642, p<.01$, but there was no significant difference between repetitions and alternations $(p>.5)$ and no significant interaction between sequence and repetition ( $p=.14$ ). These results suggest that SSRT improves on consecutive stop trials, whether or not the stop stimulus repeats.

\section{Experiment 3}

In Experiments 1 and 2, we showed that SSRT improved on consecutive stop trials. Experiment 2 suggested that this result is not stimulus specific, as the improvement was the same for repetitions and alternations of the stop stimulus. In Experiment 3, we replaced the two auditory stop signals with two visual stop signals to determine whether the repetition effects in the first two experiments are specific to auditory stop signals and whether they replicate when the go and stop stimuli are presented in the same modality.

\section{Method}

Subjects. Thirty-two subjects were selected and compensated as in Experiment 1. We replaced five subjects for accuracy below $85 \%$, two subjects for unusually slow RT ( $>3$ SDs above the mean), and one subject whose probabilities of successful stopping (calculated from those trials that contribute to the SSD tracking algorithm) fell outside the $95 \%$ confidence interval of .5 probability of stopping to a stop signal.

Apparatus and stimuli. The apparatus and stimuli were the same as in Experiment 2, with the exception that two visual stop signals (orange and blue seven-pointed stars) replaced the two auditory stop signals. Orange and blue are opposites on a standard color wheel, so their colors are as dissimilar as possible. The height and width of the stars were approximately $10 \mathrm{~cm}$ at the longest point. Unlike the go stimuli, which were filled in, these stop stimuli were only outlines with a width of approximately 0.5 $\mathrm{cm}$, in order to not overlap with the visual go stimuli.

Procedure. The basic trial structure and instructions for Experiment 3 were identical to those of Experiment 2, except visual stop signals replaced auditory stop signals.

\section{Results}

We first evaluated the results without examining sequential effects. Mean correct no-signal RT was $598 \mathrm{~ms}$, and mean nosignal accuracy was $94 \%$. Mean $p$ (respondlsignal) was .46. Additionally, $p$ (respondlsignal) did not differ significantly between the two star colors (orange $M=.46$, blue $M=.46$ ), $t(23)<1, p\rangle$ .8. We calculated $p$ (respondlsignal) for only those trials that affected the SSD tracking algorithm and found a value equal to .50 . Mean SSD was $348 \mathrm{~ms}$. The ordering of the RT distributions for signal-respond and go trials confirms the predictions of the race model (see Appendix, Figure A1c) and justifies our use of the race model to estimate SSRT.

As in previous experiments, we found post-stop-signal slowing in the go task, as S + 1 RT (603 ms) was significantly slower than $\mathrm{G}+1 \mathrm{RT}(590 \mathrm{~ms}), t(23)=2.71, p<.05$. To assess sequential effects in stopping, we calculated SSRT for the first and second stop trial in a pair in the same way we did in the Experiment 2. There was no significant difference between SSDs in repetition sequences $(M=346 \mathrm{~ms})$ and alternation sequences $(M=346 \mathrm{~ms})$, $t(23)<1, p>$.9. Mean SSRTs are presented in Figure 1c. We 
evaluated the effect of place in sequence (S1 or S2) and stop stimulus repetition or alternation on SSRT using a $2 \times 2$ ANOVA. The main effect of place in sequence was not significant, $F(1$, $31)=2.28, M S E=641, p=.14$, but this nonsignificant effect was in the direction of SSRT improving on consecutive stop trials, as S2 $\operatorname{SSRT}(M=218 \mathrm{~ms})$ was faster than $\operatorname{S1~SSRT~}(M=225 \mathrm{~ms}){ }^{2}$ There was no significant main effect of repetition or alternation $(p>.7)$ and no significant interaction $(p>.4)$. Although the effect was in the same direction as in the previous two experiments, it did not reach significance here. SSRT improvement on consecutive stop trials appears to be attenuated if both go and stop stimuli are visual. In order to test the generality of this effect, Experiment 4 used a different set of visual stop signals.

\section{Experiment 4}

The Experiment 3 results showed that when the stop stimuli are visual, SSRT decreased quantitatively on the second stop signal in a pair, but this result did not reach significance. To assess the generality of this effect further, we used a different set of visual stop signals, namely, horizontal bars presented either above or below the go stimulus.

\section{Method}

Subjects. Twenty-four subjects were selected and compensated as in Experiment 1. We replaced three subjects for mean accuracy below $85 \%$.

Apparatus and stimuli. The apparatus and stimuli were the same as in Experiment 3, with the exception that the two visual stop signals were horizontal black bars $(5 \mathrm{~cm}$ wide by $1 \mathrm{~cm}$ tall) presented either $1 \mathrm{~cm}$ above the top of the go stimulus or $1 \mathrm{~cm}$ below the bottom of the go stimulus for $100 \mathrm{~ms}$. We presented the shapes close to the go stimulus so that subjects should not need to shift eye gaze away from the center of the screen to recognize the stop signals.

Procedure. The basic trial structure and instructions for Experiment 4 were identical to those for Experiment 3, except for the changes noted in the Apparatus and stimuli section.

\section{Results}

We first evaluated the results without examining sequential effects. Mean correct no-signal RT was $582 \mathrm{~ms}$, and mean nosignal accuracy was $94 \%$. Mean $p$ (respondlsignal) was .47 . The $p$ (respondlsignal) did not differ significantly between bars presented above or below the go stimulus (above $M=.46$, below $M=.48), t(23)=1.40, p>.15$. We calculated $p($ respondlsignal $)$ for only those trials that affected the SSD tracking algorithm and found a value equal to .50. Mean SSD was $332 \mathrm{~ms}$. The ordering of the RT distributions for signal-respond and go trials confirms the predictions of the race model (see Appendix, Figure A1d) and justifies our use of the race model to estimate SSRT.

Post-stop-signal slowing did not reach significance, as S + 1 RT (585 ms) was not significantly slower than G + 1 RT (577 ms), $t(23)=1.67, p=.11$. There was no significant difference between SSDs in repetition sequences $(M=330 \mathrm{~ms})$ and alternation sequences $(M=331 \mathrm{~ms}), t(23)<1, p>$.8. To assess sequential effects in stopping, we calculated SSRT for the first and second in a pair using the method from Experiments 2 and 3. Mean SSRTs are presented in Figure 1d. We evaluated the effect of place in sequence (S1 or S2) and stop stimulus repetition or alternation on SSRT using a $2 \times 2$ ANOVA. This revealed that S2 SSRT $(M=$ $220 \mathrm{~ms})$ was faster than S1 SSRT $(M=229 \mathrm{~ms}), F(1,23)=13.96$, $M S E=144, p<.01$. There was no significant main effect of repetition or alternation $(p>.15)$ and no significant interaction $(p>.5)$.

In Experiment 3, post-stop-signal slowing was significant, but the improvement in SSRT on the second trial in a pair was not. In Experiment 4, post-stop-signal slowing was not significant, but the improvement in SSRT on the second trial in a pair was. To gain power, we did additional analyses of go RTs and SSRTs that combined the experiments. The go RT analysis was a 2 (go trial type: $\mathrm{G}+1$ or $\mathrm{S}+1) \times 2$ (Experiment: 3 or 4 ) ANOVA with go trial type as a within-subject factor and experiment as a betweensubject factor. This revealed a strong effect of go trial type, $F(1$, $54)=8.96, M S E=299, p<.01$, but no main effect of group or interaction $(p$ s $>.4)$. The SSRT analysis was a 2 (place in sequence: S1 or S2) $\times 2$ (stop stimulus: repetition or alternation) $\times 2$ (Experiment: 3 or 4 ) ANOVA, with the first two factors within group and the final factor between groups. This revealed a strong main effect of place in sequence, $F(1,54)=8.08, M S E=$ $429, p<.01$, but no other significant main effects or interactions (all $p \mathrm{~s}>.25$ ). These combined analyses suggest that visual stop signals may produce post-stop-signal slowing and post-stop-signal improvements in SSRT, but the borderline effects in each study suggest that the effects may be less robust than the effects with auditory stop signals. It is not clear whether the difference is due to the modality of the stop signal (visual vs. auditory) or the match between the modality of the stop signal and the go signal (same modality in Experiments 3 and 4; different modalities in Experiments 1 and 2).

\section{Experiment 5}

Experiments 2, 3, and 4 suggested that improved SSRT on consecutive stop trials was not stimulus specific, but stop stimulus alternations were also stop stimulus modality repetitions. In Experiments 5 and 6 , we used one auditory stop signal and one visual stop signal. If the improvement in SSRT is modality specific, then SSRT should be faster when stop-signal modality repeats than when it alternates. If the improvement in SSRT is not modality specific, then SSRT should be faster whenever a stop trial repeats, whether or not stop-signal modality repeats.

\section{Method}

Subjects. Twenty-four subjects were selected and compensated as in Experiment 1. We replaced three subjects for mean accuracy below $85 \%$.

Apparatus and stimuli. The apparatus and stimuli were the same as in Experiment 3, with the exception that the blue star was replaced with the same $500-\mathrm{Hz}$ tone used in Experiments 1 and 2.

\footnotetext{
${ }^{2}$ We ran the experiment first with 24 subjects, like the other experiments in the article, and found a marginal difference between S1 and S2 SSRT. To increase power, we increased the sample size to 32 , but the result remained marginal. We present the results with all 32 subjects.
} 
Procedure. The basic trial structure and instructions for Experiment 5 were identical to those of Experiment 3, except for the changes noted in the Apparatus and stimuli section.

\section{Results}

We first evaluated the results without examining sequential effects. Mean no-signal RT was $596 \mathrm{~ms}$, and mean no-signal accuracy was 95\%. Mean $p$ (respondlsignal) was .46. The $p$ (respondlsignal) did not differ significantly between the star and the tone (orange star $M=.45,500-\mathrm{Hz}$ tone $M=.47$ ), $t(23)<1$, $p>$.5. We calculated $p$ (respondlsignal) for only those trials that affected the SSD tracking algorithm and found a value equal to .50. Mean SSD was $340 \mathrm{~ms}$. The ordering of the RT distributions for signal-respond and go trials confirms the predictions of the race model (see Appendix, Figure Ale) and justifies our use of the race model to estimate SSRT.

We observed post-stop-signal slowing. S + 1 RT (603 ms) was slower than $\mathrm{G}+1 \mathrm{RT}(586 \mathrm{~ms}), t(23)=3.24, p<.01$. There was no significant difference between SSDs in repetition sequences $(M=343 \mathrm{~ms})$ and alternation sequences $(M=339 \mathrm{~ms}), t(23)=$ $1.02, p>$.3. Mean SSRTs, calculated as in Experiments 2, 3, and 4, are presented in Figure 1e. We evaluated the effects of place in sequence (S1 or S2) and stop stimulus repetition or alternation on SSRT using a $2 \times 2$ ANOVA (see Figure 1e). This revealed no significant main effects $(p>1)$, but the interaction was significant, $F(1,23)=15.40, M S E=258.31, p<.01$ (see Figure 1e). Planned comparisons revealed that in repetition pairs, SSRT was faster on $\mathrm{S} 2(M=215 \mathrm{~ms})$ than on $\mathrm{S} 1(M=233 \mathrm{~ms}), F(1,23)=$ $15.90, M S E=258.31, p<.01$. This was not the case in alternation pairs, in which the S2 SSRT (236 ms) was marginally slower than S1 SSRT $(229 \mathrm{~ms}), F(1,23)=2.44, M S E=258.31, p=.13$. This slowing after alternation pairs across modality is reminiscent of Bissett et al. (2009), who showed that SSRT slowed when subjects had to inhibit to a visual no-go stimulus, then inhibit to an auditory stop signal.

An alternative explanation for the difference in SSRT on the second trial in repetition or alternation pairs is that the go process is slower on the second trial in repetition pairs compared to alternation pairs. We used the same go RT distribution (i.e., S + 1 , or trials after any single stop signal) in the SSRT calculation for the second trial in repetition and alternation pairs. This calculation assumes that the repetitions or alternation of the previous stop stimulus does not impact the current trial go processing. However, it is possible that this is not the case. If repeating the stop stimulus resulted in longer go processing, $p$ (respondlsignal) would decrease, decreasing the estimate of SSRT. Therefore, the decrease in the estimate of SSRT may be a result of slower go processing after stop stimulus repetitions, not faster stop processing. To evaluate this alternative explanation, we looked at the signal-respond RTs on the second trial in repetition and alternation pairs. A slower go process on the second trial in repetitions should be reflected in slower signal-respond RTs. If the stop process speeds up, signalrespond RTs on the second trial in repetitions should be shorter, especially at slower values. To evaluate these explanations, we quantile averaged (Van Zandt, 2002; see Appendix) signal-respond RT distributions on the second trial in stop stimulus repetition and alternation pairs. Unlike in Figure A1, we used only five quantiles, as there are fewer trials in these distributions. The results reveal that signal-respond RTs on the second trial in a repetition are truncated at the later quantiles (see Figure 2a), which supports a speeding of the stop process explaining the faster SSRT, not a slowing of the go process.

These results suggest that sequential improvements in SSRT occur only when stop signals are presented in the same modality. This result also suggests that we may have observed improvement in SSRT in alternation sequences in Experiments 2, 3, and 4 because stimulus alternations were modality repetitions.

\section{Experiment 6}

Experiments 5 suggested that SSRT improved only if the modality of the stop stimulus repeats on consecutive trials. Experiment 6 presented two stop signals in different modalities but used different stimuli to test the generalizability and replicability of this effect.

\section{Method}

Subjects. Twenty-four subjects were selected and compensated as in Experiment 1. We replaced four subjects for accuracy below $85 \%$ and one subject whose probabilities of successful a Experiment 5 Signal-Respond Cumulative Distributions

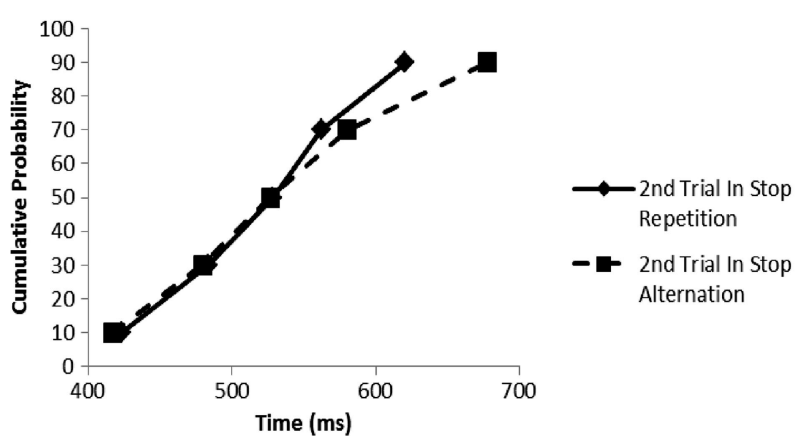

b Experiment 6 Signal-Respond Cumulative Distributions

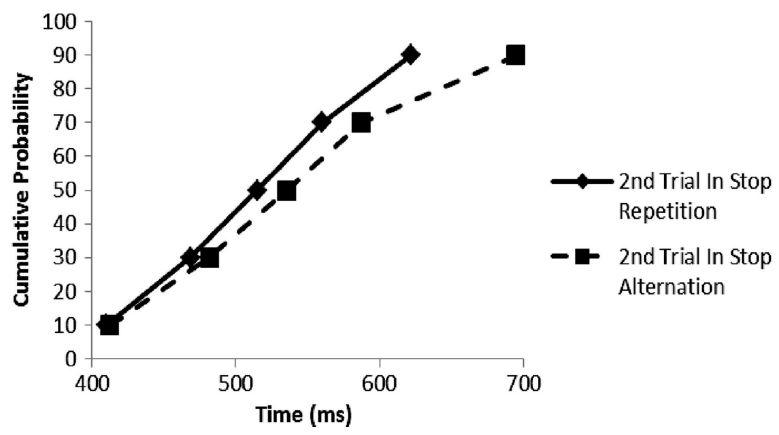

Figure 2. For Experiments 5 and 6 (Panels a and b, respectively), quantile averaged signal-respond reaction times on the second trial in pairs of stop signals, separated by whether the current stop repeats or alternates from the previous trial. 
stopping (calculated from those trials that contribute to the SSD tracking algorithm) fell outside the $95 \%$ confidence interval of .5 probability of stopping to a stop signal.

Apparatus and stimuli. The apparatus and stimuli were the same as those used in Experiments 2 and 4. The visual stop signal was always a bar either above or below the go stimulus (counterbalanced across subjects), and the auditory stop signal was always a $500-\mathrm{Hz}$ or $750-\mathrm{Hz}$ tone (counterbalanced across subjects). Each subject saw only one visual bar and heard only one auditory stop signal, and the choice of auditory and visual stop signal was counterbalanced across subjects.

Procedure. The basic trial structure and instructions for Experiment 6 were identical to those of Experiments 2 and 4, except for the changes noted in the Apparatus and stimuli section.

\section{Results}

We first evaluated the results without examining sequential effects. Mean no-signal RT was 592 ms, and mean no-signal accuracy was 95\%. Mean $p$ (respondlsignal) was .46 . We computed a 2 (modality: visual or auditory) $\times 4$ (stop-signal combination: above and $500 \mathrm{~Hz}$, above and $750 \mathrm{~Hz}$, below and $500 \mathrm{~Hz}$, below and 750 $\mathrm{Hz}$ ) ANOVA on $p$ (respondlsignal), with modality as a withinsubject factor and stop-signal combination as a between-subject factor. The ANOVA revealed a main effect of modality, with lower $p$ (respondlsignal) with auditory stop signals $(M=.44)$ than with visual stop signals $(M=.49)$ but no significant main effect of stop-signal combination or interaction of stop-signal combination with modality. We calculated $p$ (respondlsignal) for only those trials that affected the SSD tracking algorithm and found a value equal to .50 . Mean SSD was $350 \mathrm{~ms}$. The ordering of the RT distributions for signal-respond and go trials confirms the predictions of the race model (see Appendix, Figure A1f) and justifies our use of the race model to estimate SSRT.

We observed post-stop-signal slowing. S + 1 RT (598 ms) was slower than $\mathrm{G}+1 \mathrm{RT}(583 \mathrm{~ms}), t(23)=3.00, p<.01$. There was no significant difference between SSDs in repetition sequences $(M=351 \mathrm{~ms})$ and alternation sequences $(M=348 \mathrm{~ms}), t(23)<$ 1. Mean SSRTs, calculated as in Experiments 2-5, are presented in Figure 1f. We evaluated the effect of place in sequence (S1 or S2) and stop stimulus repetition or alternation on SSRT using a $2 \times 2$ ANOVA (see Figure 1f). This revealed a main effect of repetition or alternation, $F(1,23)=4.44, M S E=794, p<.05$; no significant main effect of place in sequence, $F(1,23)<1$; but a strong interaction, $F(1,23)=12.35, M S E=353, p<.01$. Figure if shows that the main effect is driven entirely by the second trial in the pair. Planned comparisons revealed that in repetition pairs, SSRT was faster on S2 $(M=198 \mathrm{~ms})$ than on S1 $(M=215 \mathrm{~ms})$, $F(1,23)=9.25, M S E=353, p<.01$. The slowing in alternation pairs was borderline significant, with S2 SSRT (224 ms) longer than S1 SSRT $(213 \mathrm{~ms}), F(1,23)=3.72, M S E=353, p=.07$. This interaction of place in pair with repetition or alternation replicates Experiment 5, using different visual stop signals and an additional auditory tone.

As in Experiment 5, we wanted to ensure that the difference in SSRT on the second trial in repetition and alternations pairs is driven by adjustments in the speed of the stop process, not the go process. We ran the same analysis on these data, and a similar result was revealed (Figure $2 b$ ), showing that signal-respond RTs are shorter on the second trial in stop repetitions, especially at the later quantiles. This provides strong support that adjustments of the stop process are driving the differences in SSRT, not adjustments of the go process.

\section{General Discussion}

Slowing of go RT after a stop signal is commonly observed (Bissett \& Logan, 2011a; Emeric et al., 2007; Rieger \& Gauggel, 1999; Verbruggen et al., 2008). Here we examined whether this commonly observed shift away from the go task (slower go RT) results in a shift toward the stop task (faster SSRT). In the auditory stop-signal experiments ( 1 and 2$)$ and when we combined the results for the two visual stop-signal experiments (3 and 4), we showed that SSRT improved after stop trials. Additionally, we assessed whether SSRT improvements were stimulus specific. SSRT improved if the stop stimulus repeated or if the stop stimulus alternated within the same modality, but SSRT did not improve if the stop stimulus changed modality (see Experiments 5 and 6). This reveals modality-specific poststop-signal speeding of SSRT.

These stimulus-specific results cannot be explained by the similarity between stimuli. In Experiments 2, 3, and 4, the two stop stimuli were very different frequencies, opposite colors on a color wheel, or presented in different locations, respectively, but SSRT improved as much when these dissimilar stimuli alternated across trials as when identical stimuli repeated.

The current results provide some insight into previous investigations of the interaction between successive acts of inhibition. These experiments show that successive performance of the same stop task with stop stimuli in the same modality speeds SSRT, consistent with Morein-Zamir et al. (2007). Experiments 5 and 6 show that stop signals in different modalities eliminate the speeding of SSRT and may produce a modest slowing of SSRT. Other studies that have shown slowing of SSRT on consecutive inhibition trials have used two different inhibitory tasks, instead of consecutive stop trial performance (Bissett et al., 2009; van den Wildenberg et al., 2002; Verbruggen et al., 2004, 2005). This suggests that behaviorally, a change in inhibitory task has a similar effect to a change in stop-signal modality, namely, eliminating or reversing any SSRT improvement.

\section{Are Stop-Signal Repetition Effects Automatic or Strategic?}

Adjustments of go RT and SSRT following stop signals could be strategic or automatic. Strategies involve an explicit choice between alternative ways of doing a task (Logan, 1985). Strategies are flexible and can be implemented immediately and proactively, based on instructions, cues, or explicit knowledge. By contrast, automatic processes do not involve explicit choice. They are relatively inflexible and are driven by bottom-up stimulus processing rather than instructions, cues, or explicit knowledge (Logan, 1988). Bissett and Logan (2011a) evaluated several explanations of post-stop-signal slowing of go RT and decided that a strategic adjustment - a shift in goal priority away from the go taskexplained the data best. This shift in goal priority appears to be affected by probabilities, payoffs, and motivation, and tradeoffs appear to be aimed at maximizing utility (Bissett \& Logan, 2011a, 
2011b; Leotti \& Wager, 2010; Liddle et al., 2009; Padmala \& Pessoa, 2010; Shenoy, Rao, \& Yu, 2010; Wong-Lin, Eckhoff, Holmes, \& Cohen, 2010). Bissett and Logan (2011b) manipulated conditional probabilities of stop signals and found greatly increased post-stopsignal slowing when subjects expected stop signals but eliminated or reversed post-stop-signal slowing when subjects did not expect stop signals. These large adjustments occurred immediately following cues, suggesting that post-stop-signal slowing was dominated by strategies, not reflexes or implicit learning.

It is tempting to interpret the present evidence for post-stop-signal speeding of SSRT as the strategic counterpart of post-stop-signal slowing, reflecting a shift in goal priority toward the stop task. However, the modality specificity of the SSRT improvement urges caution in yielding to that temptation. There was greater speeding when the modality of the stop signal repeated than when it alternated in Experiments 5 and 6. This modality specificity is reminiscent of stimulus-specific repetition priming effects, which result from automatic processing (Logan, 1988, 1990; Soetens, Boer, \& Hueting, 1985). Further research will be needed to determine whether poststop-signal speeding of SSRT is strategic or automatic, or both.

\section{Implications for Major Cognitive Control Frameworks}

In the introduction, we outlined three major cognitive control frameworks: error monitoring (Laming: 1968; Rabbitt, 1966), conflict monitoring (Botvinick et al., 2001; Carter et al., 1998), and episodic memory retrieval (Tulving, 1972; Verbruggen \& Logan, 2008a, 2008b). The current results are not consistent with error monitoring. Error monitoring suggests that subjects increase their response threshold after errors, lengthening RT and reducing errors (Laming, 1968; Rabbitt, 1966). When applied to the stop-signal task, error monitoring would suggest that subjects increase their response threshold for the go task, producing post-stop-signal slowing (Bissett \& Logan, 2011a). However, error monitoring might also increase the threshold for choosing to stop, which should slow SSRT. We observe the opposite, speeding of SSRT.

The current results could be consistent with the conflict monitoring hypothesis, which claims that control is recruited after conflict trials to increase the likelihood of achieving task goals. The go stimulus and the stop signal could create conflict on a stop-signal trial, and that might lead to an adjustment of go and stop processes that makes stopping more likely. The adjustment to the go process could be an increase in response threshold that would slow go RT and increase the likelihood that the stop process would beat the go process (Logan \& Cowan, 1984; but see Bissett \& Logan, 2011a). The adjustment to the stop process might be a reduction in stop response threshold to speed SSRT and increase likelihood that the stop process would beat the go process, as we observed. However, to account for our results, the conflict hypothesis would have to explain why conflict is greater when stop-signal modality repeats than when it alternates. We see no ready explanation.

The results here are not consistent with most theories of episodic memory retrieval, as similarity of successive stop stimuli did not affect post-stop-signal SSRT improvement (Logan, 1988, 1990; Palmeri, 1997). In Experiments 2-4, stop trial alternations were two dissimilar stop stimuli presented in the same modality, and the improvement in SSRT on these alter- nations did not differ from exact repetitions of the stop stimulus. In Experiments 5-6, alternations were two dissimilar stop stimuli presented in different modalities, and this revealed no post-stop-signal SSRT improvements on alternations, but the SSRT improvement in repetitions remained. These results suggest speeding of SSRT depends on the repetition of the modality of the stop signal, not the similarity of successive stop signals. This is consistent with theories that assume that repetition benefits depend on activating the same processing pathway (Posner \& Snyder, 1975).

Episodic retrieval accounts also predict that retrieval should facilitate performance most when there is an exact match between the events on the previous trial and the current trial (Altmann, 2011; Barsalou, 1990; Hommel, 1997; Hommel et al., 2004; Logan, 1988, 1990; Mayr et al., 2003; Medin \& Schaffer, 1978; Neill \& Valdes, 1992; Neill et al., 1992; Nosofsky, 1984; Tulving, 1972; Verbruggen \& Logan, 2008a, 2008b). We did not have sufficient power to isolate trials with the same go stimuli, stop stimuli, and response, but we could exclude trials with the same go and stop stimuli and investigate the remaining trials, ensuring that our results were not driven by exact repetitions of both go and stop stimuli. The SSRT results without complete repetitions looked virtually identical to those including complete repetitions, suggesting that the improvement in SSRT on modality repetitions is not driven by complete repetitions of the previous episode.

\section{Conclusions}

The present research shows that SSRT is faster when a stop signal in the same modality appeared on the previous trial. These effects extend previous research on post-stop-signal adjustment that focused on slowing of go RT following stop signals, suggesting that the two effects may be different sides of the same coin, reflecting a shift in goal priority away from the go task toward the stop task (Bissett \& Logan, 2011a, 2011b).

\section{References}

Altmann, E. M. (2011). Testing probability matching and episodic retrieval accounts of response repetition effects in task switching. Journal of Experimental Psychology: Learning, Memory, and Cognition, 37, 935951. doi:10.1037/a0022931

Barsalou, L. W. (1990). On the indistinguishability of exemplar memory and abstraction in category representation. In T. K. Srull \& R. S. Wyer (Eds.), Advances in social cognition: Volume III. Content and process specificity in the effects of prior experiences (pp. 61-88). Hillsdale, NJ: Erlbaum.

Bissett, P. G., \& Logan, G. D. (2011a). Balancing cognitive demands: Control adjustments in the stop-signal paradigm. Journal of Experimental Psychology: Learning, Memory, and Cognition, 37, 392-404. doi: $10.1037 / \mathrm{a} 0021800$

Bissett, P. G., \& Logan, G. D. (2011b). Post-stop-signal slowing: Strategies dominate reflexes and implicit learning. Journal of Experimental Psychology: Human Perception and Performance. Advance online publication. doi:10.1037/a0025429

Bissett, P. G., Nee, D. E., \& Jonides, J. (2009). Dissociating interferencecontrol processes between memory and response. Journal of Experimental Psychology: Learning, Memory, and Cognition, 35, 1306-1316. doi:10.1037/a0016537

Botvinick, M. M., Braver, T., Barch, D., Carter, C., \& Cohen, J. (2001). 
Conflict monitoring and cognitive control. Psychological Review, 108, 624-652. doi:10.1037/0033-295X.108.3.624

Carter, C. S., Braver, T. S., Barch, D. M., Botvinick, M. M., Noll, D., \& Cohen, J. D. (1998, May 1). Anterior cingulate cortex, error detection, and the online monitoring of performance. Science, 280, 747-749. doi:10.1126/science.280.5364.747

Emeric, E. E., Brown, J. W., Boucher, L., Carpenter, R. H. S., Hanes, D. P., Harris, R., . . Schall, J. D. (2007). Influence of history on countermanding saccade performance in humans and macaque monkeys. Vision Research, 47, 35-49. doi:10.1016/j.visres.2006.08.032

Gehring, W. J., Goss, B., Coles, M. G. H., Meyer, D. E., \& Donchin, E. (1993). A neural system for error detection and compensation. Psychological Science, 4, 385-390. doi:10.1111/j.1467-9280.1993.tb00586.x

Hajcak, G., \& Simmons, R. F. (2008). Oops! I did it again: An EROP and behavioral study of double errors. Brain and Cognition, 68, 15-21. doi:10.1016/j.bandc.2008.02.118

Hommel, B. (1997). Interactions between stimulus-stimulus congruence and stimulus-response compatibility. Psychological Research, 59, 248260. doi:10.1007/BF00439302

Hommel, B., Proctor, R. W., \& Vu, K.-P. L. (2004). A feature-integration account of sequential effects in the Simon task. Psychological Research, 68, 1-17. doi:10.1007/s00426-003-0132-y

Ito, S., Stuphorn, V., Brown, J. W., \& Schall, J. D. (2003, October 3). Performance monitoring by the anterior cingulate cortex during saccade countermanding. Science, 302, 120-122. doi:10.1126/science.1087847

Kühn, S., \& Brass, M. (2009). When doing nothing is an option: The neural correlates of deciding whether to act or not. Neurolmage, 46, 11871193. doi:10.1016/j.neuroimage.2009.03.020

Kühn, S., Elsner, B., Prinz, W., \& Brass, M. (2009). Busy doing nothing: Evidence for nonaction-effect binding. Psychonomic Bulletin \& Review, 16, 542-549. doi:10.3758/PBR.16.3.542

Laming, D. (1968). Information theory of choice-reaction times. London, England: Academic Press.

Lappin, J. S., \& Eriksen, C. W. (1966). Use of a delayed signal to stop a visual reaction-time response. Journal of Experimental Psychology, 72, 805-811. doi:10.1037/h0021266

Lenartowicz, A., Verbruggen, F., Logan, G. D., \& Poldrack, R. A. (2011). Inhibition-related activation in the right inferior frontal gyrus in the absence of inhibitory cues. Journal of Cognitive Neuroscience, 23, 3388-3399. doi:10.1162/jocn_a_00031

Leotti, L. A., \& Wager, T. D. (2010). Motivational influences on response inhibition measures. Journal of Experimental Psychology: Human Perception and Performance, 36, 430-447. doi:10.1037/a0016802

Levitt, H. (1971). Transformed up-down method in psychoacoustics. Journal of the Acoustical Society of America, 49, 467-477. doi:10.1121/ 1.1912375

Liddle, E. B., Scerif, G., Hollis, C. P., Batty, M. J., Groom, M. J., Liotti, M., \& Liddle, P. F. (2009). Looking before you leap: A theory of motivated control of action. Cognition, 112, 141-158. doi:10.1016/ j.cognition.2009.03.006

Logan, G. D. (1985). Executive control of thought and action. Acta Psychologica, 60, 193-210. doi:10.1016/0001-6918(85)90055-1

Logan, G. D. (1988). Toward an instance theory of automatization. Psychological Review, 95, 492-527. doi:10.1037/0033-295X.95.4.492

Logan, G. D. (1990). Repetition priming and automaticity: Common underlying mechanisms. Cognitive Psychology, 22, 1-35. doi:10.1016/ 0010-0285(90)90002-L

Logan, G. D., \& Cowan, W. B. (1984). On the ability to inhibit thought and action: A theory of an act of control. Psychological Review, 91, 295327. doi:10.1037/0033-295X.91.3.295

Logan, G. D., Schachar, R. J., \& Tannock, R. (1997). Impulsivity and inhibitory control. Psychological Science, 8, 60-64. doi:10.1111/j.14679280.1997.tb00545.x
Mayr, U., Awh, E., \& Laurey, P. (2003). Conflict adaptation effects in the absence of executive control. Nature Neuroscience, 6, 450-452.

Medin, D. L., \& Schaffer, M. M. (1978). Context theory of classification learning. Psychological Review, 85, 207-238. doi:10.1037/0033295X.85.3.207

Morein-Zamir, S., Chua, R., Franks, I., Nagelkerke, P., \& Kingstone, A. (2007). Predictability influences stopping and response control. Journal of Experimental Psychology: Human Perception and Performance, 33, 149-162. doi:10.1037/0096-1523.33.1.149

Neill, W. T., \& Valdes, L. A. (1992). The persistence of negative priming Steady-state or decay? Journal of Experimental Psychology: Learning, Memory, and Cognition, 18, 565-576. doi:10.1037/0278-7393.18.3.565

Neill, W. T., Valdes, L. A., Terry, K. M., \& Gorfein, D. S. (1992) Persistence in negative priming: II. Evidence for episodic trace retrieval Journal of Experimental Psychology: Learning, Memory, and Cognition, 18, 993-1000. doi:10.1037/0278-7393.18.5.993

Nelson, M. J., Boucher, L., Logan, G. D., Palmeri, T. J., \& Schall, J. D. (2010). Nonindependent and nonstationary response times in stopping and stepping saccade tasks. Attention, Perception, \& Psychophysics, 72, 1913-1929. doi:10.3758/APP.72.7.1913

Nosofsky, R. M. (1984). Choice, similarity, and the context theory of classification. Journal of Experimental Psychology: Learning, Memory, and Cognition, 10, 104-114. doi:10.1037/0278-7393.10.1.104

Padmala, S., \& Pessoa, L. (2010). Interactions between cognition and motivation during response inhibition. Neuropsychologia, 48, 558-565. doi:10.1016/j.neuropsychologia.2009.10.017

Palmeri, T. J. (1997). Exemplar similarity and the development of automaticity. Journal of Experimental Psychology: Learning, Memory, and Cognition, 23, 324-354. doi:10.1037/0278-7393.23.2.324

Posner, M. I., \& Snyder, C. R. R. (1975). Attention and cognitive control. In R. Solso (Ed.), Information processing and cognition: The Loyola Symposium (pp. 205-223). Hillsdale, NJ: Erlbaum.

Rabbitt, P. M. (1966). Errors and error correction in choice-response tasks. Journal of Experimental Psychology, 71, 264-272. doi:10.1037/ h0022853

Rabbitt, P. M., \& Rodgers, B. (1977). What does a man do after he makes an error? An analysis of response programming. The Quarterly Journal of Experimental Psychology, 29, 727-743. doi:10.1080/14640747708400645

Rieger, M., \& Gauggel, S. (1999). Inhibitory after-effects in the stop signal paradigm. British Journal of Psychology, 90, 509-518. doi:10.1348/ 000712699161585

Schachar, R. J., Chen, S., Logan, G. D., Ornstein, T. J., Crosbie, J., Ickowicz, A., \& Pakulak, A. (2004). Evidence for an error monitoring deficit in attention deficit hyperactivity disorder. Journal of Abnormal Child Psychology, 32, 285-293. doi:10.1023/B:JACP $0000026142.11217 . f 2$

Shenoy, P., Rao, R., \& Yu, A. J. (2010). A rational decision making framework for inhibitory control. In J. Lafferty, C. K. I. Williams, J. Shawe-Taylor, R. S. Zemel, \& A. Culotta (Eds.), Advances in neural information processing systems (Vol. 23, pp. 2146-2154). Cambridge, MA: MIT Press.

Shower, E. G., \& Biddulph, R. (1931). Differential pitch sensitivity of the ear. Journal of the Acoustical Society of America, 3, 275-287. doi $10.1121 / 1.1915561$

Soetens, E., Boer, L. C., \& Hueting, J. E. (1985). Expectancy or automatic facilitation? Separating sequential effects in two-choice reaction time Journal of Experimental Psychology: Human Perception and Performance, 11, 598-616. doi:10.1037/0096-1523.11.5.598

Stuphorn, V., \& Schall, J. D. (2006). Executive control of countermanding saccades by the supplementary eye field. Nature Neuroscience, 9, 925931. doi:10.1038/nn1714

Stuphorn, V., Taylor, T. L., \& Schall, J. D. (2000, December 14). Performance monitoring by the supplementary eye field. Nature, 408, 857860. doi: $10.1038 / 35048576$ 
Tulving, E. (1972). Episodic and semantic memory. In E. Tulving \& W. Donaldson (Eds.), Organization of memory (pp. 381-402). New York, NY: Academic Press

van den Wildenberg, W. P. M., van der Molen, M. W., \& Logan, G. D. (2002). Reduced response readiness delays stop signal inhibition. Acta Psychologica, 111, 155-169. doi:10.1016/S0001-6918(02)00020-3

Van Zandt, T. (2002). Analysis of response time distributions. In J. T. Wixted \& H. Pashler (Eds.) Steven's Handbook of Experimental Psychology (3rd Edition), Volume 4: Methodology in Experimental Psychology (pp. 461-516). New York: Wiley Press.

Verbruggen, F., Liefooghe, B., Notebaert, W., \& Vandierendonck, A. (2005). The effects of stimulus-stimulus compatibility and stimulusresponse compatibility on response inhibition. Acta Psychologica, 120, 307-326. doi:10.1016/j.actpsy.2005.05.003

Verbruggen, F., Liefooghe, B., \& Vandierendonck, A. (2004). The interaction between stop signal inhibition and distractor interference. Acta Psychologica, 116, 21-37. doi:10.1016/j.actpsy.2003.12.011

Verbruggen, F., \& Logan, G. D. (2008a). Automatic and controlled response inhibition: Associative learning in the go/no-go and stop-signal paradigms. Journal of Experimental Psychology: General, 137, 649672. doi:10.1037/a0013170

Verbruggen, F., \& Logan, G. D. (2008b). Long-term aftereffects of response inhibition: Memory retrieval task goals and cognitive control Journal of Experimental Psychology: Human Perception and Performance, 34, 1229-1235. doi:10.1037/0096-1523.34.5.1229

Verbruggen, F., Logan, G. D., Liefooghe, B., \& Vandierendonck, A. (2008). Short-term aftereffects of response inhibition: Repetition priming or between-trial control adjustment? Journal of Experimental Psychology: Human Perception and Performance, 34, 413-426. doi: 10.1037/0096-1523.34.2.413

Wever, E. G., \& Wedell, C. H. (1941). Pitch discrimination at high frequencies. Psychological Bulletin, 38, 727.

Wong-Lin, K., Eckhoff, P., Holmes, P., \& Cohen, J. D. (2010). Optimal performance in a countermanding saccade task. Brain Research, 1318, 178-187. doi:10.1016/j.brainres.2009.12.018

Zwicker, E., \& Fastl, H. (1990). Psychoacoustics: Facts and models. Berlin, Germany: Springer-Verlag.

\section{Appendix}

\section{Testing the Race Model}

To justify the use of the race model (Logan \& Cowan, 1984) to estimate SSRT in all six experiments, we wanted to test the fundamental prediction of the race model that signal-respond RTs should be shorter than RTs for all no-signal trials. Not only should they be faster, but the fastest signal-respond RTs should be similar to the fastest no-signal RTs, and the distribution of reaction times should diverge at their longer RTs, with the longest signal-respond RTs being considerably faster than the longest no-signal RTs. This prediction is derived from the fact that signal-respond trials are stop trials that escape inhibition, and therefore should disproportionately reflect the fastest part of the no-signal RT distribution, as the long tail should be truncated because of successful inhibition of slow go activation (Logan \& Cowan, 1984).

To evaluate this prediction, we plotted cumulative distribution functions for signal-respond RTs and all no-signal RTs. In order to retain the shape of each subject's RT distribution, we quantile averaged the group RTs (Van Zandt, 2002). Quantile averaging involves rank ordering each subject's RTs and selecting a set number of RTs from this rank ordering that correspond to the RTs of different quantiles. For example, in this case, we selected the 10 RTs that correspond to the 5 th, 15 th, 25th, 35th, 45th, 55th, 65th, 75th, 85th, and 95th percentiles of each subject's RT distributions (separately for no-signal RTs and signal-respond RTs). We then separately averaged each quantile across subjects, revealing one ultimate quantile average of no-signal RTs and signal-respond RTs. These are presented in Figure A1, Panels a-f. These results support the predictions of the race model, as signal-respond RTs are faster than no-signal RTs, and the difference is largest at the later quantiles, in all six experiments. 
a. Experiment 1 Cumulative Distributions

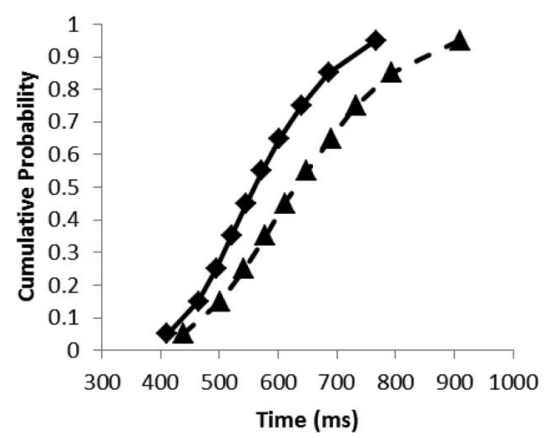

c. Experiment 3 Cumulative Distributions

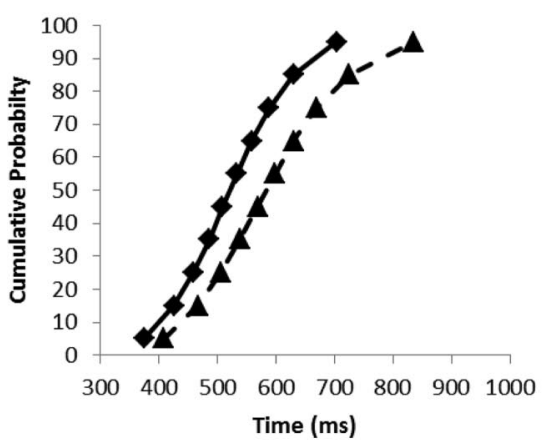

e. Experiment 5 Cumulative Distributions

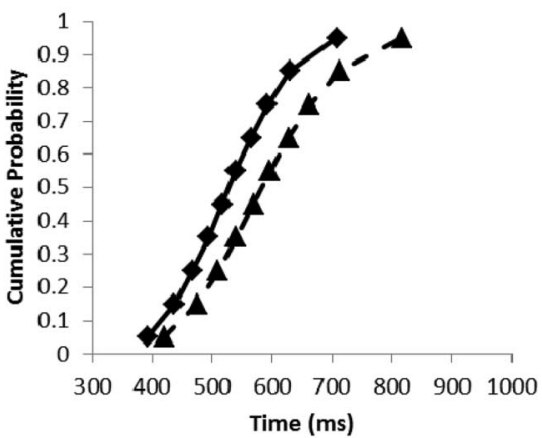

b. Experiment 2 Cumulative Dlstrlbutions

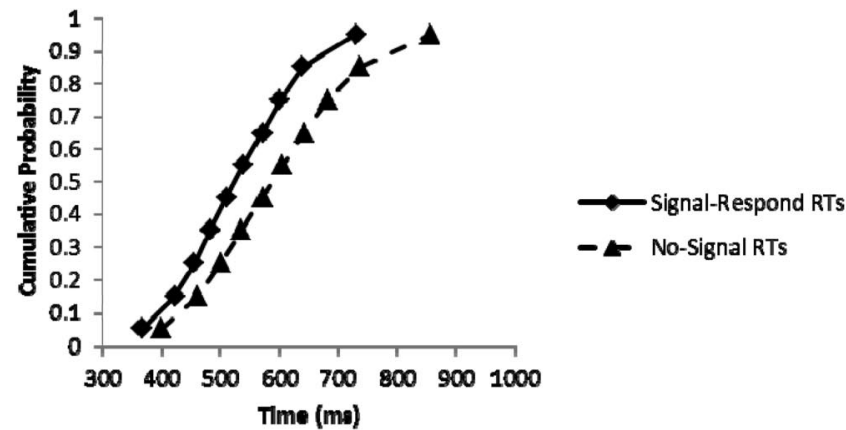

d. Experiment 4 Cumulative Distributions

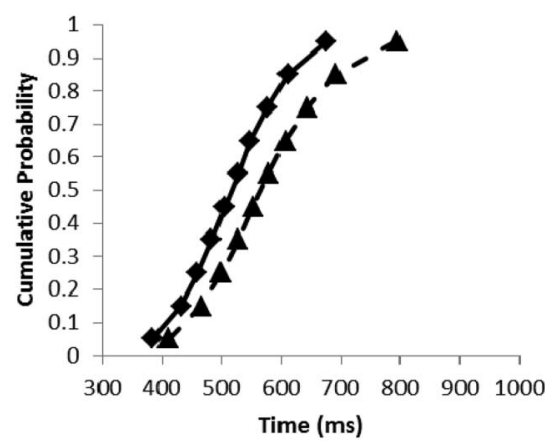

f. Experiment 6 Cumulative Distributions

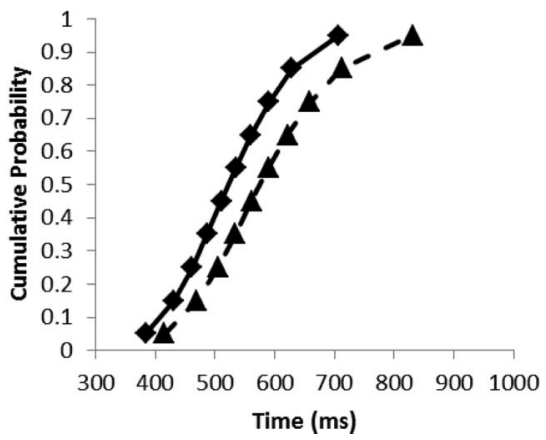

$\longrightarrow$ Signal-Respond RTs $\sim$ - No-Signal RTs $\longrightarrow$ Signal-Respond RTs

- \& No-Signal RTs

$\multimap$ Signal-Respond RTs

$\iota$ No-Signal RTs

Received February 14, 2011

Revision received November 15, 2011

Accepted November 15, 2011 